\title{
A NOTE ON THE METHOD OF MULTIPLE SCALES*
}

\author{
BY GEORGE VERONIS (Yale University)
}

\begin{abstract}
A modification of the method of multiple scales makes use of the expansion of parameters of the system in order to remove undesirable characteristics of the solution obtained by the usual multiple-scale method. When applied to the damped harmonic oscillator, the modification leads to the exact solution. For the Duffing equation it leads to an approximation which can be reduced to the solution reported by Nayfeh [1]. However, the solution derived here appears to be more accurate and the frequency takes on a form without nonuniformities.

The method of multiple scales produces a uniformly valid expansion for systems in which a troublesome term is multiplied by a small parameter and for which an ordinary perturbation expansion leads to a nonuniformly valid series solution. For example, the weakly damped harmonic oscillator

$$
\ddot{x}+2 \varepsilon \dot{x}+\omega^{2} x=0, \quad \varepsilon \ll 1,
$$

has the general solution

$$
x=\left[a_{0} e^{i \sqrt{\omega^{2}-\varepsilon^{2}} t}+a_{0}^{*} e^{-i \sqrt{\omega^{2}-\varepsilon^{2}} t}\right] e^{-\varepsilon t},
$$

where $a_{0}^{*}$ is the complex conjugate of $a_{0}$. An ordinary perturbation in $\varepsilon$ yields results which correspond to expanding $e^{-\varepsilon t}$ and $\exp \left[ \pm i\left(\omega^{2}-\varepsilon^{2}\right)^{1 / 2} t\right.$ in power series which are nonuniformly valid since $t$ can always be large enough to offset the smallness of $\varepsilon$ or $\varepsilon^{2}$.

The derivative expansion method of multiple scales [1, pp. 236-240] makes use of the time expansion

$$
t_{n}=\varepsilon^{n t}, \quad x=x\left(t_{0}, t_{1}, t_{2} \cdots\right), \quad d x / d t=D_{0} x+\varepsilon D_{1} x+\varepsilon^{2} D_{2} x+\cdots,
$$

where $D_{n} \equiv \partial / \partial t_{n}$. The time $t_{0}$ is the unstretched time coordinate and the remaining $t_{n}$ correspond to longer time scales. To $O\left(\varepsilon^{2}\right)$ the solution

$$
\begin{aligned}
x & =A e^{-t_{1}} \exp \left[i \omega\left(t_{0}-\frac{1}{2 \omega^{2}} t_{2}\right)\right] \\
& =A e^{-\varepsilon t} \exp \left[i \omega\left(t-\varepsilon^{2} t / 2 \omega^{2}\right)\right]+C C,
\end{aligned}
$$

where $C C$ stands for complex conjugate, is obtained by Nayfeh [1] with the derivativeexpansion method, the two-variable expansion method and the generalized method of multiple scales.

It is clear that (4) is a distinct improvement over the ordinary perturbation solution since the important exponentially decaying part of the solution emerges and the amplitude $A$ does not involve the time. However, it is also clear that the solution still contains a secular type of behavior since the frequency term is not uniformly valid. The fact that

* Received January 9, 1980. This work was supported by NSF grant OCE-77-19451. 
the essential physics is already contained in (4) is gratifying, but that is not a feature that can generally be obtained for more complicated problems. Hence, it will be helpful to eliminate the inadequacy in (4) if that is possible.

In this example it is obvious that, since the lowest-order equation is

$$
D_{0}^{2} x+\omega^{2} x=0,
$$

the lowest-order solution is proportional to $\exp \left[ \pm i \omega t_{0}\right]$ and subsequent orders can only modify this result. Since the inadequacy is caused by the "wrong" lowest-order frequency, it is plausible to try to correct the lowest-order frequency. We do so by expanding the coefficient of the last term in (1), viz. the parameter $\omega^{2}$, in powers of $\varepsilon$ by writing

$$
\omega^{2}=\omega_{0}^{2}+\varepsilon \omega_{1}+\varepsilon^{2} \omega_{2}+\cdots
$$

where the first term is written as a square for convenience. At the outset none of the $\omega_{n}$ are known. We shall evaluate them by imposing the condition that the frequency be nonsecular, thereby eliminating the inadequacy in (4).

Making use of (3) and (6) in (1), we obtain

$$
\begin{aligned}
{\left[D_{0}^{2}+2 \varepsilon D_{1} D_{0}+\varepsilon^{2}\left(D_{1}^{2}+2 D_{0} D_{2}\right)+\cdots\right] x+\omega_{0}^{2} x } \\
=-2 \varepsilon\left(D_{0}+\varepsilon D_{1}+\varepsilon^{2} D_{2}+\cdots\right) x-\varepsilon \omega_{1} x-\varepsilon^{2} \omega_{2} x-\cdots .
\end{aligned}
$$

Then equating to zero the coefficients of like powers of $\varepsilon$ yields to $O\left(\varepsilon^{2}\right)$

$$
\begin{gathered}
D_{0}^{2} x+\omega_{0}^{2} x=0, \\
2 D_{0} D_{1} x=-2 D_{0} x-\omega_{1} x, \\
\left(D_{1}^{2}+2 D_{0} D_{2}\right) x=-2 D_{1} x-\omega_{2} x .
\end{gathered}
$$

The general solution of $(8)$ is

$$
x=A\left(t_{1}, t_{2}\right) \exp \left[i \omega_{0} t_{0}\right]+C C .
$$

Substituting (11) into (9) yields

$$
\left(2 i \omega_{0} D_{1} A+2 i \omega_{0} A+\omega_{1} A\right) \exp \left[i \omega_{0} t_{0}\right]+C C=0
$$

and, since the coefficients of $\exp \left[i \omega_{0} t_{0}\right]$ and $\exp \left[-i \omega_{0} t_{0}\right]$ must vanish separately,

$$
D_{1} A+\left(1-\frac{i \omega_{1}}{2 \omega_{0}}\right) A=0
$$

and

$$
A=a\left(t_{2}\right) \exp \left[-t_{1}\left(1-\frac{i \omega_{1}}{2 \omega_{0}}\right)\right] .
$$

As pointed out earlier, the coefficient $\omega_{1}$ is to be evaluated so that the frequency is nonsecular. Hence,

$$
\omega_{1}=0, \quad A=a\left(t_{2}\right) e^{-t_{1}} .
$$

Substituting (11) and (14) into (10) yields

$$
\left[2 i \omega_{0} D_{2} a+\left(\omega_{2}-1\right) a\right] \exp \left[-t_{1}+i \omega_{0} t_{0}\right]+C C=0 .
$$


Hence,

$$
D_{2} a-\frac{i\left(\omega_{2}-1\right)}{2 \omega_{0}} a=0
$$

and

$$
a=a_{0} \exp \left[i\left(\omega_{2}-1\right) t_{2} / 2 \omega_{0}\right] .
$$

In order to suppress secular terms in the frequency we must have

$$
\omega_{2}=1, \quad a=a_{0} .
$$

Hence, to $O\left(\varepsilon^{2}\right)$ the solution is

$$
x=a_{0} \exp \left[i \omega_{0} t_{0}-t_{1}\right]+C C
$$

with

$$
\omega^{2}=\omega_{0}^{2}+\varepsilon^{2} \quad \text { or } \quad \omega_{0}^{2}=\omega^{2}-\varepsilon^{2} .
$$

Therefore, the solution (20) can be written

$$
x=a_{0} \exp \left[i \sqrt{\omega^{2}-\varepsilon^{2}} t-\varepsilon t\right]+C C
$$

which is identical to (2).

The fact that the exact solution emerges here is fortuitous. However, the reasoning is applicable to more complicated problems for which the exact solution cannot be obtained, and it yields results that are at least an improvement over the usual methods of multiple scales. In each case, the procedure is equivalent: expand the available parameters in the equation(s) and use the unknown coefficients of the expansion to remove undesirable traits of the approximate solution. To a certain extent the choice of undesirable traits that one removes may be subjective. In the foregoing example there was only one inadequacy, the secular frequency, so the choice was obvious.

A second example is the Duffing equation

$$
\ddot{u}+\omega^{2} u+\varepsilon u^{3}=0
$$

with a nonlinear spring described by the term $\varepsilon u^{3}$. To $O\left(\varepsilon^{2}\right)$ the solution obtained by Nayfeh [1] by the three multiple-scale methods is

$$
u=a \cos (\bar{\omega} t+\chi)+\frac{\varepsilon a^{3}}{32 \omega^{2}}\left(1-\frac{21 a^{2} \varepsilon}{32 \omega^{2}}\right) \cos 3(\bar{\omega} t+\chi)+\frac{a^{5} \varepsilon^{2}}{1024 \omega^{4}} \cos 5(\bar{\omega} t+\chi),
$$

where

$$
\bar{\omega}=\omega+\frac{3 a^{2} \varepsilon}{8 \omega}-\frac{150^{4} \varepsilon^{2}}{256 \omega^{2}} .
$$

Here again, the frequency contains secular terms which limit the validity of the solution, so we shall use (6) to suppress this secularity since the lowest-order frequency arises from the coefficient of $u$ in (23).

Because of the nonlinearity in this problem it is necessary to expand $u$ in a series of $\varepsilon$ as well,

$$
u=\sum_{n=0}^{N} \varepsilon^{n} u_{n}
$$


Then with expansions equivalent to (3) we obtain the equations to $O\left(\varepsilon^{2}\right)$

$$
\begin{gathered}
D_{0}^{2} u_{0}+\omega_{0}^{2} u_{0}=0, \\
D_{0}^{2} u_{1}+\omega_{0}^{2} u_{1}=-2 D_{0} D_{1} u_{0}-u_{0}^{3}-\omega_{1} u_{0}, \\
D_{0}^{2} u_{2}+\omega_{0}^{2} u_{2}=-2 D_{0} D_{1} u_{1}-2 D_{0} D_{2} u_{0}-D_{1}^{2} u_{0}-3 u_{0}^{2} u_{1}-\omega_{1} u_{1}-\omega_{2} u_{0} .
\end{gathered}
$$

Eq. (27) has the solution

$$
u_{0}=A\left(t_{1}, t_{2}\right) \exp \left[i \omega_{0} t_{0}\right]+C C
$$

and $(28)$ becomes

$D_{0}^{2} u_{1}+\omega_{0}^{2} u_{1}=\left(-2 i \omega_{0} D_{1} A-\omega_{1} A-3 A^{2} A^{*}\right) \exp \left[i \omega_{0} t_{0}\right]-A^{3} \exp \left[3 i \omega_{0} t_{0}\right]+C C$.

To suppress secular terms of the form $t_{0} \exp \left[i \omega_{0} t_{0}\right]$ we set the coefficient of $\exp \left[i \omega_{0} t_{0}\right]$ on the right-hand side equal to zero:

$$
2 i \omega_{0} D_{1} A+\omega_{1} A+3 A^{2} A^{*}=0 .
$$

Now write

$$
A=\frac{a}{2} e^{i \phi}
$$

where $a\left(t_{1}, t_{2}\right)$ and $\phi\left(t_{1}, t_{2}\right)$ are real. Then equating real and imaginary parts of $(32)$, we obtain

$$
D_{1} a=0, \quad D_{1} \phi=\frac{\omega_{1}}{2 \omega_{0}}+\frac{3 a^{2}}{8 \omega_{0}}
$$

with solutions

$$
a=a\left(t_{2}\right), \quad \phi=\left(\frac{\omega_{1}}{2 \omega_{0}}+\frac{3 a^{2}}{8 \omega_{0}}\right) t_{1}+\phi_{0}\left(t_{2}\right)
$$

so that

$$
A=\frac{a\left(t_{2}\right)}{2} \exp \left[i\left(\frac{\omega_{1}}{2 \omega_{0}}+\frac{3 a^{2}}{8 \omega_{0}}\right) t_{1}+i \phi_{0}\left(t_{2}\right)\right]
$$

To suppress secular terms in the frequency, we require

$$
\omega_{1}=-3 a^{2} / 4
$$

so that $D_{1} A=0$ and

$$
A=\frac{a\left(t_{2}\right)}{2} \exp \left[i \phi_{0}\left(t_{2}\right)\right]
$$

The remaining terms in (31) lead to the solution

$$
u_{1}=B\left(t_{1}, t_{2}\right) \exp \left[i \omega_{0} t_{0}\right]+\frac{A^{3}}{8 \omega_{0}^{2}} \exp \left[3 i \omega_{0} t_{0}\right]+C C \text {. }
$$


With (30), (37), (38) and (39) the right-hand side of (29) becomes

$$
\begin{aligned}
\left\{-2 i \omega_{0}\left(D_{1} B+\right.\right. & \left.\left.D_{2} A\right)-3\left(A^{2} B^{*}+2 A A^{*} B+\frac{A^{* 2} A^{3}}{8 \omega_{0}^{2}}\right)-\omega_{2} A-\omega_{1} B\right\} \exp \left[i \omega_{0} t_{0}\right] \\
& -\left\{\left(3 A^{2} B+\frac{3 A^{4} A^{*}}{4 \omega_{0}^{2}}+\frac{\omega_{1} A^{3}}{8 \omega_{0}^{2}}\right) \exp \left[3 i \omega_{0} t_{0}\right]+\frac{3 A^{5}}{8 \omega_{0}^{2}} \exp \left[5 i \omega_{0} t_{0}\right]\right\}+C C .
\end{aligned}
$$

The coefficient of $\exp \left[i \omega_{0} t_{0}\right]$ must vanish if secular terms are to be eliminated. This is accomplished by writing $B \equiv 0$ and with $A$ given by (38), we obtain

$$
D_{2} a=0, \quad \omega_{0} D_{2} \phi_{0}=\frac{3 a^{5}}{256 \omega_{0}^{2}}+\frac{\omega_{2} a}{2} .
$$

Thus, $a$ is a constant and in order to suppress secular behavior in frequency we must have $D_{2}\left(\phi_{0}\right)=0$ or

$$
\omega_{2}=-3 a^{4} / 128 \omega_{0}^{2} .
$$

The terms multiplying $\exp \left[3 i \omega_{0} t_{0}\right]$ and $\exp \left[5 i \omega_{0} t_{0}\right]$ in (40) yield

$$
u_{2}=\frac{a^{5}}{2048 \omega_{0}^{4}} \exp \left[5 i\left(\omega_{0} t_{0}+\phi_{0}\right)\right]+\frac{3 a^{5}}{2048 \omega_{0}^{4}} \exp \left[3 i\left(\omega_{0} t_{0}+\phi_{0}\right)\right] \text {. }
$$

Hence, the solution of $(23)$ to $O\left(\varepsilon^{2}\right)$ is

$$
\begin{aligned}
u=a\left[\cos \left(\omega_{0} t+\phi_{0}\right)+\frac{\varepsilon a^{2}}{32 \omega_{0}^{2}}\left(1+\frac{3 \varepsilon a^{2}}{32 \omega_{0}^{2}}\right) \cos 3\left(\omega_{0} t_{0}\right.\right. & \left.+\phi_{0}\right) \\
& \left.+\left(\frac{\varepsilon a^{2}}{32 \omega_{0}^{2}}\right) \cos 5\left(\omega_{0} t_{0}+\phi_{0}\right)\right],
\end{aligned}
$$

where

$$
\omega^{2}=\omega_{0}^{2}-\frac{3 a^{2}}{4} \varepsilon\left(1+\frac{a^{2} \varepsilon}{32 \omega_{0}^{2}}\right) .
$$

Eq. (45) can be solved for $\omega_{0}$. To this order it suffices to set $\omega_{0}^{2}=\omega^{2}$ in the last term and obtain

$$
\omega_{0}=\left(\omega^{2}+\frac{3 a^{2} \varepsilon}{4}\left(1+\frac{a^{2} \varepsilon}{32 \omega^{2}}\right)\right)^{1 / 2} .
$$

This solution reduces to (24) if $\omega_{0}$ is expanded in powers of $\varepsilon$ and the expansion is substituted into (44) wherever $\omega_{0}$ appears. However, the present form is more accurate in the sense that it contains (naturally) selected higher-order terms in the expression for $\omega_{0}$. Furthermore, the frequency $\omega_{0}$ is expressed in a uniformly valid form rather than as a power series of terms multiplying $t$. The solution is still valid only to $O\left(\varepsilon^{2}\right)$ but the present form may be more useful, especially if one uses it to evaluate $u$ for values of $\varepsilon$ that are not so small.

In the two examples discussed above the unknown coefficients in the expansion (6) are used to suppress secularity in the frequency. In other problems the coefficients may 
be used for removing other undesirable traits of the approximate solution. The suggested procedure is to carry out the usual multiple-scales analysis, find where the inadequacies

(if any) of the approximate solution occur and then use expansions analogous to (6) to remove those inadequacies.

\section{REFERENCE}

[1] A. H. Nayfeh, Perturbation methods, Wiley-Interscience, John Wiley and Sons, New York, 1973 